\title{
Gamma ray emission from magnetized relativistic GRB outflows
}

\section{Andrii Neronov and Volodymyr Savchenko}

ISDC Data Center for Astrophysics, Chemin d'Ecogia 16, 1290 Versoix, Switzerland and Geneva Observatory, 51 ch. des Maillettes, CH-1290 Sauverny, Switzerland

E-mail: Andrii.Neronov@unige.ch, Volodymyr.Savchenko@unige.ch

\begin{abstract}
We argue that small pitch angle synchrotron emission provides an important dissipation mechanism that has to be taken into account in models of the formation of relativistic magnetized $\gamma$-ray burst (GRB) outflows from newborn black holes and/or magnetars. We show that if the GRB outflow is proton loaded, the spectral energy distribution of this emission is expected to sharply peak in the 0.1-1 MeV energy band. If the small pitch-angle synchrotron emission efficiently cools relativistic particles of the outflow, the emission spectrum below the peak energy is a power law with spectral index $\alpha \simeq-1$, close to the typical spectral index of time-resolved GRB spectra. Otherwise, the low energy spectral index can be as hard as $\alpha \simeq 0$, as observed at the beginning of the GRB pulses. We conjecture that small pitch-angle synchrotron emission from proton-loaded magnetized GRB outflow could significantly contribute to the Band component of the prompt emission of GRBs, while electromagnetic cascade initiated by the protons may be responsible for the GeV component.
\end{abstract}

25th Texas Symposium on Relativistic Astrophysics - TEXAS 2010

December 06-10, 2010

Heidelberg, Germany 


\section{Introduction}

A large amount of information about the time evolution of the spectral characteristics of prompt emission of $\gamma$-ray bursts (GRB) has been collected by CGRO/BATSE, Swift/BAT, INTEGRAL/ISGRI (see [22] and [25] for reviews) and, the Fermi/GBM [6] telescopes. It is firmly established that the majority of the time-resolved GRB spectra could be well described by relatively simple spectral models, such as the Band model [5], or a cut-off or broken power-law model determined by three main parameters: low and high energy photon indices $\alpha$ and $\beta$, and peak (or break or cut-off) energy $E_{p}[25,22]$.

The observed distribution of the low-energy power-law indices $\alpha$ (of differential photon spectra given by $d N_{\gamma} / d E \sim E^{\alpha}$ ) of time-resolved GRB spectra is sharply peaked at the value $\alpha \simeq-1$ in the BATSE GRB [5, 15] and Swift/BAT [28] GRB samples. Deviations from this mean value toward $\alpha>-1$ (down to $\alpha \simeq 0$ ) are preferentially observed at the initial moments of onset of GRB sub-pulses, when the spectra could be as hard as $\alpha \gtrsim 0[26,15,28]$. Evolution toward $\alpha<-1$ is commonly observed during the decay of individual sub-pulses and/or at the end of the prompt GRB emission phase. The characteristic value $\alpha \simeq-1$ is difficult to reconcile with the conventionally assumed synchrotron and/or inverse Compton mechanisms of prompt GRB emission. Alternative models, which attempt to resolve this problem, include "jitter" radiation [20, 21] (see [18] for a discussion of the potential problems of the jitter radiation model) and "quasi thermal comptonization" [11] models.

Spectra of at least some GRBs contain a separate GeV-band component (e.g, [1, 7]). The GeV emission has a different (relative to the Band component) temporal evolution [12]. The absence of the high-energy cutoff in the spectrum of this $\mathrm{GeV}$ component due to the pair-production imposes a lower bound on the bulk Lorentz factors of the GRB outflows (about $\Gamma \gtrsim 1000$, e.g. [4], [1]). Different models of the origin of the $\mathrm{GeV}$ emission have been proposed, such as high-energy extention of the conventional afterglow [17, 13], afterglow emission from a fireball in the radiative regime [12], and synchrotron [27] or cascade [9, 3] emission from ultra high-energy $\left(\sim 10^{20} \mathrm{eV}\right)$ protons.

Several models of the formation of relativistic GRB outflows from black holes or neutron stars, formed in result of core collapse of massive stars, assume that the outflow is highly magnetized $[16,8]$. In these models, the energy of the outflow is initially carried by the Poynting flux of electromagnetic energy. Numerical MHD calculations show that the electromagnetic energy could be converted in a dissipationless way to the kinetic energy of relativistic particle outflow. Dissipation is absent because the electromagnetic field forms a force-free configuration.

In what follows we show that even if electromagnetic field is force-free, the energy loss of relativistic particles in the outflow is non-zero, because the directions of the velocities of individual particles are scattered around the average direction of the flow within a certain angle $\Psi_{0} \sim \Gamma_{0}^{-1}$, where $\Gamma_{0}$ is the Lorentz factor of the outflow. This scatter in particle velocities leads to radiative dissipation of the outflow energy, the main dissipation mechanism being small pitch-angle synchrotron emission. We demonstrate that a significant fraction of the power of magnetized outflow could be dissipated by this mechanism even in the outflow acceleration region.

We derive the spectral characteristics of this emission and show that they are consistent with those of the observed GRB prompt emission spectra. 


\section{Generic properties of pre-shock relativistic GRB outflow}

If the observed isotropic luminosity of GRB is $L_{\text {iso }}$, the energy flux carried by the GRB outflow ejected into a solid angle $\Omega$ is $L=(\Omega / 4 \pi) L_{\text {iso }} \simeq 8 \times 10^{47}\left[L_{\text {iso }} / 10^{52} \mathrm{erg} / \mathrm{s}\right]\left[\Theta / 1^{\circ}\right]^{2} \mathrm{erg} / \mathrm{s}$, where $\Theta$ is the opening angle of the outflow. The energy density of the outflow at a distance $D$ is $U=$ $L /\left(\Omega D^{2}\right)^{1}$. It is convenient to parametrize the strength of the electromagnetic field in the outflow in terms of magnetization parameter $\sigma, B^{2} /(8 \pi) \sim \sigma U /(1+\sigma)$. Using this a parametrization, the (electro)magnetic field strength at the distance $D$ can be expressed as $B=\sqrt{2 \sigma L_{\text {iso }} /(1+\sigma)} / D$.

The minimal possible angular scatter in the velocities of the particles is within a cone of opening angle $\Psi_{0} \simeq \kappa \Gamma_{0}^{-1}$ ( $\kappa$ is a numerical factor of the order of 1). Although the radiation, discussed below, is leading to decrease of $\Psi_{0}$, the minimal scatter corresponding $\kappa \sim 1$ holds as long as the acceleration process is active. This misalignment gives birth to a non-zero Lorentz force of the order of $F \sim e B_{\perp}$, where $e$ is particle charge and $B_{\perp} \sim B \Psi_{0}=\kappa B \Gamma_{0}^{-1}$. Particle motions due to this uncompensated Lorentz force lead to the radiation, by specificly to the small pitch angle synchrotron emission, which possesses the proporties significantly different from those of synchrotron emission from an isotropic particle distribution [10, 19]. The typical energy of the radiated photons, $\varepsilon_{s} \sim e B_{\perp} \Gamma_{0}^{2} / m_{p}$, where $m_{p}$ is the particle mass (proton mass in the above and in the following numerical estimates), scales proportionally to the particle energy, rather than to the square of it. The synchrotron energy loss rate $P_{s}=2 e^{4} B_{\perp}^{2} \Gamma_{0}^{2} /\left(3 m_{p}^{2}\right) \sim \kappa^{2} B^{2}$ remarkably does not depend on the particle energies $\Gamma_{0} m_{p}$.

To estimate the importance of small pitch angle synchrotron cooling as a dissipation mechanism, one has to compare the synchrotron cooling distance $D_{s}=\Gamma_{0} m_{p} / P_{s}$ with the typical distance scales of formation and propagation of the GRB outflow. Equating $D_{s}$ to $D$ one can find that synchrotron emission can efficiently remove energy from relativistic protons out to the distance $\mathscr{D}_{s} \simeq 1.3 \times 10^{9} \kappa^{2}\left[\frac{\sigma}{1+\sigma}\right]\left[\frac{L_{\text {iso }}}{10^{52} \mathrm{ergs} / \mathrm{s}}\right]\left[\frac{\Gamma_{0}}{10^{4}}\right]^{-1} \mathrm{~cm}$. The typical distance scale of the GRB central engine $R_{\mathrm{CE}} \sim 10^{6}-10^{7} \mathrm{~cm}$ is given by the size of black hole formed in the collapse of a massive star, or the radius of the magnetar. Both $R_{\mathrm{CE}}$ and $D_{\Gamma}$ might be $\lesssim \mathscr{D}_{s}$ implying that small pitch-angle synchrotron emission could be an efficient dissipation mechanism. Similarly one finds that the electrons, do not contribute significantly to the total energy dissipation in the distance range of the interest.

One other condition to be fulfilled is that the emission region has to optically thin with respect to Compton scattering. One can easily find that this is the case starting from the distance $\mathscr{D}_{\mathrm{C}} \simeq$ $5 \times 10^{6}\left[\frac{L_{\text {iso }}}{10^{52} \mathrm{erg} / \mathrm{s}}\right]\left[\frac{\Gamma_{0}}{10^{4}}\right]^{-3} \mathrm{~cm}$. In GRBs with bulk Lorentz factors $\Gamma_{0} \geq 6 \times 10^{2}, \mathscr{D}_{\mathrm{C}}<\mathscr{D}_{s}$ and synchrotron photons freely escape if they are produced in the distance range $\mathscr{D}_{\mathrm{C}}<D<\mathscr{D}_{\text {s }}$.

\section{Spectrum of emission from pre-shock proton loaded GRB outflow}

This type of spectrum could be calculated by summing the spectra emitted from the outflow components moving at different angles $\theta$ with respect to the line of sight. A non-negligible contribution is given only by $\theta$ in the range $0<\theta<\left(\Psi_{0}+\Gamma^{-1}\right)$. Ommiting the calculation details, presented in [23], we note that the required flux density can be expressed in the following form:

\footnotetext{
${ }^{1}$ We use natural system of units $c=1$.
} 


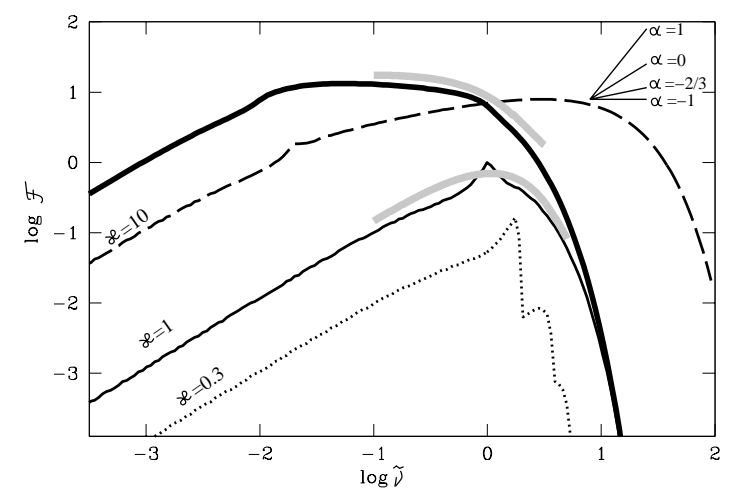

Figure 1: Spectra of small pitch-angle proton synchrotron emission from pre-shock GRB outflow for several values of $\kappa$. Thick solid line shows an emission spectrum from a proton spectrum formed by cooling of the outflow protons to $\Gamma=0.01 \Gamma_{0}$. For comparison, we show Band model fits to the spectra of periods "b" (beginning) and "d+e" (end) of Fermi GRB 090902B [7] as lower and upper grew curves. Normalizations and peak energies of the Band model fits to GRB 090902B spectra are shifted to match the small pitch-angle synchrotron model curves.

$$
F_{\Gamma_{0}}(v)=\frac{4 \pi e^{2} v_{B}}{\kappa^{2} \Gamma_{0}} \mathscr{F}(\tilde{v}, \kappa),
$$

where $\tilde{v}=v /\left(2 \Gamma_{0} v_{B}\right)$ and $\mathscr{F}(\tilde{v}, \kappa)$ is a function that depends on neither the magnetic field strength nor the Lorentz factor. Numerically calculated functions $\mathscr{F}(\tilde{V}, \kappa)$ are shown for several values of $\kappa$ in Fig. 1. At low frequencies, $\tilde{v} \ll 1$, the function $\mathscr{F}_{\gamma}(\tilde{v}, \kappa)$ has the form of a hard spectral index power-law, $\mathscr{F} \gamma(\tilde{v}, \kappa) \sim \tilde{v}^{\alpha+1}$ with $\alpha=0$. The spectrum becomes even harder close to the Doppler shifted cyclotron frequency $\tilde{v}=1$. If $\kappa<1$, the function $\mathscr{F}(\tilde{v}, \kappa)$ has more-orless strong higher harmonics of the cyclotron line, which can be seen as multiple "bumps" in the spectrum at $\tilde{v} \geq 1$.

\section{High-energy emission from the "tail" of angular distribution}

The spectrum marked $\kappa=1$ in Fig. 1 is calculated based on the assumption that the angular distribution of particle velocities, $N_{p}(\Psi)$, is cut-off at the angle $\Psi_{0} \simeq \Gamma_{0}^{-1}$. In general, the spectrum of emission from protons moving at large pitch angles $\kappa \gg 1$ extends up to the frequency $\tilde{v} \simeq \kappa$, while the frequency below which the spectrum is characterized by $\alpha=0$ moves down to $\tilde{v} \simeq \kappa^{-2}$ (see Fig. 1). In the range $\kappa^{-2} \tilde{v}<\kappa$, the spectrum is characterized by a photon index $\alpha=-2 / 3$ typical of synchrotron radiation from an isotropic particle distribution (Fig. 1). If the large pitch angle tail of $N_{p}(\Psi)$ has the form of a power-law, $N_{p}\left(\kappa \Gamma_{0}^{-1}\right) \sim \kappa^{-s}$, the spectrum of emission above the characteristic (peak) frequency $v=2 \Gamma_{0} v_{B}$ has the form of a power-law with photon index $\beta=s-1$. Then the angular distribution extend to $\Psi \sim 1$ the emission spectrum reaches as far as $\tilde{v} \sim \Gamma_{0}$, or up to the $1-10 \mathrm{GeV}$ band if $\Gamma_{0} \sim 10^{4}$. The presence of the large angle tails of proton angular distribution could, therefore, be responsible for the appearance of high-energy extensions of the prompt GRB spectra similar to that observed in GRB 080916C [1]. 


\section{Emission from a distribution of protons formed by the small pitch angle synchrotron cooling}

The total proton energy-loss $P_{\text {synch }}\left(\Gamma_{0}\right)$ rate could be found by integrating of $F_{\Gamma_{0}}(v)$. Since it does not depend on the Lorentz factor $\Gamma_{0}$, a low-energy tail of particle spectrum below the initial proton energy $\Gamma_{0} m_{p}$ is generated. Stationary distribution given the continious monochromatic particle injection with $\Gamma=\Gamma_{0}$, the spectrum of the synchrotron-cooled protons $N_{p}(\Gamma)$ is given by a power law with the slope $b=0$ in the range of Lorentz factors $\Gamma \leq \Gamma_{0}$. The corresponding spectrum of small pitch-angle synchrotron emission is

$$
F(v)=\int N_{p}(\Gamma) F_{\Gamma}(v) d \Gamma \sim v^{\alpha+1}, \alpha=-(b+1)=-1 .
$$

The overall spectral evolution of the pre-shock outflow emission is expected to be as follows. As soon as synchrotron cooling of the outflow particles becomes efficient, the spectrum of emission from the pre-shock outflow softens from $\alpha \simeq 0$ ( or $\alpha=-2 / 3$, depending on the initial value of $\kappa$ ) down to $\alpha \simeq-1$. The $\alpha \simeq-1$ spectrum persists over a period of stable activity of the GRB central engine as long as synchrotron cooling remains the dominant energy-loss channel. This value of $\alpha$ is close to the measured characteristic low-energy spectral index of CGRO/BATSE and Swift/BAT GRBs $[15,28]$. Prompt emission spectra of some GRBs start from very low values of $\alpha>-2 / 3$ $[26,15,28]$ and evolve toward $\alpha \simeq-1$ as expected if the very hard emission originates from the pre-shock GRB outflow. As an example, we show in Fig. 1 Band model fits to the spectra of the beginning and end of the prompt emission phase of the GeV- $\gamma$-ray-loud GRB 090902B, taken from [7]. Comparing the Band model fit of the initial rising phase of GRB 090902B with the spectrum of emission from monochromatic proton distribution, one could conclude that GRB 090902B outflow is initially almost monoenergetic. One can derive initial $\Gamma_{0} \sim 10^{4}$, i.e. proton energy $\Gamma_{0} m_{p} \sim$ $10^{13} \mathrm{eV}$, given the isotropic luminosity $E_{\text {iso }} \sim 10^{53} \mathrm{erg} / \mathrm{s}$ and peak energy $E_{\text {peak }} \sim \varepsilon_{s} \sim 10^{6} \mathrm{eV}$ for this particular GRB.

Small pitch-angle synchrotron emission leads not only to cooling but also to scattering in the proton beam across a wider angle $\Psi \sim \Gamma^{-1} \gg \Gamma_{0}^{-1}$. The widening of the opening angle of particle distribution could lead to the "switching on" of interactions between the particles of the outflow. It is straightforward to estimate that the mean free path $\lambda_{p p}$ becomes comparable or shorter than $\mathscr{D}_{s}$ as soon as synchrotron cooling leads to the appearance of protons with energies $\Gamma \lesssim 10^{-2} \Gamma_{0}$ if $\Gamma_{0} \sim 10^{4}$.

Development of proton initiated cascade leads to injection of secondary electrons/positrons (as $\gamma$-rays and well as neutrinos) which can largely outnumber the primary electrons present in the unshocked GRB outflow. In constrast to the primary electrons, synchrotron emission from these cascade electrons could provide a significant energy dissipation mechanism of the outflow. Although typical initial Lorentz factors of the secondary electrons are $\gamma_{e} \sim\left(\Gamma m_{p}\right) / m_{e} \gg \Gamma_{0}$, electrons are injected at the typical pitch angles $\psi_{e} \sim \Gamma^{-1} \gg \gamma_{e}^{-1}$, so that the synchrotron emission from the cascade electrons is not emitted in the small pitch angle regime.

The $\gamma$-rays with energies above the pair production threshold $E_{\gamma \gamma}=\sqrt{2 m_{e}^{2} c^{4} /(1-\cos (1 / \Gamma))} \simeq$ $10^{10}\left[\Gamma / 10^{4}\right] \mathrm{eV}$ produce pairs in interactions among themselves and initiate the electromagnetic cascade. The highest energy $\gamma$-rays may also interact with protons via pion and pair production channels. The energy loss of protons due to the $p p$ (and/or $p \gamma)$ interactions is given by 
$P_{p p}\left(\Gamma_{0}\right) \sim f \Gamma_{0} / \lambda_{p p}$, where $f \sim 0.1-0.2$ is the typical inelasticity of $p p$ collisions. As soon as the cooling of the highest energy protons by means of $p p$ and $p \gamma$ interactions becomes more efficient than the synchrotron cooling, the spectrum of protons is expected to soften to $b>0$. This should lead to the softening of the small pitch angle synchrotron emission spectrum to $\alpha<-1$ and a decrease in its contribution to the overall GRB spectrum, compared to the contribution from the cascade emission component. The spectrum of emission from the proton-initiated cascade extends up to the energy of the threshold of the pair production, $E_{\gamma \gamma}$. For the particular example of GRB 090902B, the electromagnetic cascade component is then clearly identified as the soft emission component extending up to the multi-GeV energies, which becomes dominant at the end of the prompt emission phase and persists for $\sim 10^{3} \mathrm{~s}$ after the end of the prompt emission [7].

Bursts that initiate with very hard spectra would be the most interesting candidates for testing the hypothesis of small pitch-angle proton synchrotron emission because they might possess the cyclotron line at the frequency $v \sim \Gamma_{0} v_{B}$. We search for this line at the energy close to the peak energy of very hard GRB spectra to test the proposed model. The cyclotron line feature may, however, have been "washed out" of the spectrum by a non-negligible spread in the particle energies in the outflow and by small angle Compton scattering if most of the small pitch-angle synchrotron emission is generated at the distances $D \lesssim \mathscr{D}_{\mathrm{C}}$. In this case, the spectrum of emission from the preshock outflow would be indistinguishable from the generic Band model spectra. A complementary way of testing the small pitch angle proton synchrotron model is to search for the appearance of neutrino signal from $p p$ interactions [24] at the moment of softening of the spectrum of prompt emission to $\alpha \leq 1 \mathrm{in} \mathrm{GeV} \gamma$-ray-loud GRBs. The neutrino signal is expected to be sharply peaked at energies $E_{v} \sim f \Gamma_{0} m_{p} \simeq 1\left[\Gamma_{0} / 10^{4}\right] \mathrm{TeV}$ and its flux is expected to be comparable to the luminosity of the cascade $(\mathrm{GeV})$ component of the GRB spectrum. The peak energy of neutrino signal can be predicted if the bulk Lorentz factor $\Gamma_{0}$ is estimated from the measurement of the peak energy of the proton synchrotron componenr. A search for the neutrino counterparts of the $\mathrm{GeV} \gamma$-ray loud GRBs becomes possible after a cross-correlation of the signal of Fermi/LAT-detected GRBs with the $\mathrm{TeV}$ neutrino signal detected bu the $\mathrm{km}^{3}$ scale neutrino telescope IceCube, which will become possible in the nearest future [2].

\section{Summary}

We have explored the possibility that small pitch-angle proton synchrotron emission from the magnetized GRB outflow gives significant contribution to the GRB spectrum. This emission provides an important dissipation mechanism in the region of acceleration of GRB outflows with high magnetization parameter $\sigma$. We have shown that the steady-state spectrum of this emission is expected to have photon index $\alpha=-1$, close to the characteristic photon index of the timeresolved GRB spectra. A small pitch-angle proton synchrotron emission component could also explain the extremely hard spectra $\alpha \gtrsim 0$ observed at the beginning of some GRBs. The possibility that small pitch angle proton synchrotron emission from the region of acceleration of GRB outflow could be identified in the observed GRB spectra implies that the models of formation of magnetized relativistic outflow by newly born stellar mass black holes or magnetars could be observationally tested. Whether a small pitch angle synchrotron emission component exists in the GRB spectra 
could be verified by searching for the cyclotron line features in spectra of (some of the) hardest GRBs and/or searching for prompt $\mathrm{TeV}$ neutrino emission from $\mathrm{GeV} \gamma$-ray-loud GRBs.

\section{Acknowledgment}

We would like to thank A.Taylor for discussions of the subject. The work of AN is supported by the Swiss National Science Foundation grant PP00P2_123426.

\section{References}

[1] Adbo A.A. et al. (Fermi collaboration), 2009, Science, 323, 1688.

[2] Abbasi R. et al. (IceCube collaboration), 2009, Ap.J., 701, L47.

[3] Katsuaki Asano, Sylvain Guiriec, and Peter Meszaros., 2009, Ap.J., 705L, 191A

[4] Baring, M. G. and Harding, A. K., 1997, Ap.J., 491, L663.

[5] Band D. et al., 1993, Ap.J., 413, 281.

[6] Band D. et al., 2009, Ap.J., 701, 1673.

[7] Bissaldi E., et al., 2009, arXiv:0909.2470.

[8] Bucciantini N., Quataert E., Metzger B.D., Thompson T.A., Arons J., Del Zanna L., 2009, MNRAS, 396, 2038.

[9] Charles D. Dermer and Armen Atoyan., 2006, N.J.Ph., 8, 122D

[10] Epstein R.J., 1973, Ap.J., 183, 593.

[11] Ghisellini G., Celotti A., 1999, Ap.J., 511, L93.

[12] G. Ghisellini, G. Ghirlanda, L. Nava, and A. Celotti., 2010, MNRAS 403, 926.

[13] G. Ghirlanda, G. Ghisellini, and L. Nava., 2010, A\&A., 510, 7.

[14] Ginzburg V.L., Syrovatskii S.I., 1969, ARA\&A, 7, 375.

[15] Kaneko Y., et al., 2006, Ap.J. Supp., 166, 298.

[16] Komissarov S.S., Vlahakis N., Königl A., Barkov M.V., 2010, MNRAS 394, 1182.

[17] Pawan Kumar and Rodolfo Barniol Duran., 2009, MNRAS 400, 75.

[18] Kirk J.G., Reville B., 2010, Ap.J., 710, L16.

[19] Lloyd N.M., Petrosian V., 2000, Ap.J., 543, 722.

[20] Medvedev M., Loeb S., 1999, Ap.J., 526, 697.

[21] Medvedev M., 2006, Ap.J., 637, 869.

[22] Mészáros P., 2006, Rep. Prog. Phys. 69, 2259.

[23] Neronov A., Savchenko V., 2010, A\&A, 520L, 1

[24] Paczynski B., Xu G., 1994, Ap.J., 427, 708.

[25] Piran T., 2004, Rev. Mod. Phys., 76, 1143.

[26] Preece R.D. et al., 2000, Ap.J., 506, L23.

[27] Soebur Razzaque, Charles D. Dermer, and Justin D. Finke., 2009, arXiv:1004.3330v2.

[28] Savchenko V., Neronov A., 2009, MNRAS, 396, 935. 